\title{
A Case of Homozygous Disruption of P450 Side- Chain Cleavage (CYP11A1): Cerebral MRI and CSF Neurotransmitter Findings
}

\author{
Seza Bolat ${ }^{\mathrm{a}}$, Thomas Eckey ${ }^{\mathrm{b}}$, Olaf Hiort ${ }^{\mathrm{c}}$, Andreas Moser ${ }^{\mathrm{a}, \mathrm{d}}$, e
}

\begin{abstract}
Mitochondrial cytochrome P450 side-chain cleavage enzyme (P450scc, CYP11A1) is the initiating enzyme of steroidogenesis and converts cholesterol to pregnenolone. Here we describe neurological, cerebral magnetic resonance imaging (MRI), and cerebrospinal fluid (CSF) findings in a patient with a homozygous disruption of P450scc. Free $\gamma$-aminobutyric acid (GABA) and glutamate levels were measured in the patient's CSF and control samples from 10 subjects by mean of high performance liquid chromatography with electrochemical detection. In the CSF, patient's free GABA levels were significantly reduced in comparison to control values. Cerebral MRI revealed multiple supratentorial located T2 lesions in the periventricular and peritrigonal white matter without gadolinium enhancement or diffusion restriction of lesions. Both MRI and CSF neurotransmitter findings in our patient with P450scc deficiency were comparable to those found in multiple sclerosis.
\end{abstract}

Keywords: P450 side-chain cleavage enzyme; Cerebrospinal fluid; MRI; Multiple sclerosis; $\gamma$-aminobutyric acid

\section{Introduction}

Mitochondrial cytochrome P450 side-chain cleavage enzyme (P450scc) is encoded by a single-copy gene on chromosome 15 , is the initiating enzyme of steroidogenesis and converts cholesterol to pregnenolone. For a long time, it was thought that deficiency of P450scc (CYP11A1) was not compatible with

Manuscript submitted February 5, 2018, accepted March 15, 2018

aDepartment of Neurology, University of Luebeck, Luebeck, Germany bInstitute of Neuroradiology, University of Luebeck, Luebeck, Germany 'Department of Pediatrics, Division of Pediatric Endocrinology and Diabetes, University of Luebeck, Luebeck, Germany

${ }^{\mathrm{d}}$ Freiburg Institute for Advanced Studies (FRIAS), University of Freiburg, Freiburg, Germany

${ }^{\mathrm{e}}$ Corresponding Author: Andreas Moser, Department of Neurology, University of Luebeck, Ratzeburger Allee 160, D-23538 Luebeck, Germany.

Email: andreas.moser@neuro.uni-luebeck.de

doi: https://doi.org/10.14740/jnr467w life due to lack of progesterone for maintenance of pregnancy. However, recently a small number of patients with missense or even nonsense mutations of CYP11A1 have been described [1]. Here we describe neurological, cerebral magnetic resonance imaging (MRI), and cerebrospinal fluid (CSF) findings in a patient with a homozygous disruption of P450scc with prematurity, complete $46, \mathrm{XY}$ sex reversal, hypergonadotropic hypogonadism, and primary adrenal insufficiency.

\section{Case Report}

A 19-year-old patient suffered from intermittent, several minutes lasting paresthesia of hands and feet for 6 months. Furthermore, she reported on blurred or double vision for a couple of seconds. The symptoms usually occurred during periods of increased mental or physical pressure and were of high variability. In the medical history, she had been born prematurely after 31 weeks of gestation and had severe primary adrenal insufficiency and a completely female phenotype despite $46, \mathrm{XY}$ karyotype. The gonads were small testes and she had no Mullerian structures. She received typical replacement therapy with hydrocortisone and fludrocortisone, as well as estrogen monotherapy for induction of puberty. During childhood and adolescence, several episodes of adrenal crisis occurred. Homogenous disruption of the CYP11A1 gene was diagnosed with complete loss of steroidogenesis [2].

Subsequently, she was presented to our hospital since cerebral MRI suspected multiple sclerosis (MS). Neurological examinations were completely normal. Somatosensory and visual evoked potentials were within normal limits. Serological analyses showed no signs for vasculitis, collagenosis, or mixed connective tissue disease. The serum vitamin levels for B1, B12, and folic acid were in normal range. Lumbar puncture revealed 3 cells $/ \mu \mathrm{L}$, normal $\mathrm{CSF} /$ serum albumin ratio, without intrathecal Ig-synthesis and without oligoclonal IgGbands. Serum cholesterol and LDL-cholesterol were slightly enhanced to $5.1 \mathrm{mmol} / \mathrm{L}(<5.0)$ and $3.11 \mathrm{mmol} / \mathrm{L}(<3.01)$, respectively. During the following weeks, symptoms spontaneously disappeared, and MRI findings were stable 6 months later. Although the MRI findings were, however, remarkable, a reliable relationship between reported short lasting, varying symptoms and MRI alterations could not be established. Therefore, we assumed a somatoform disorder. 


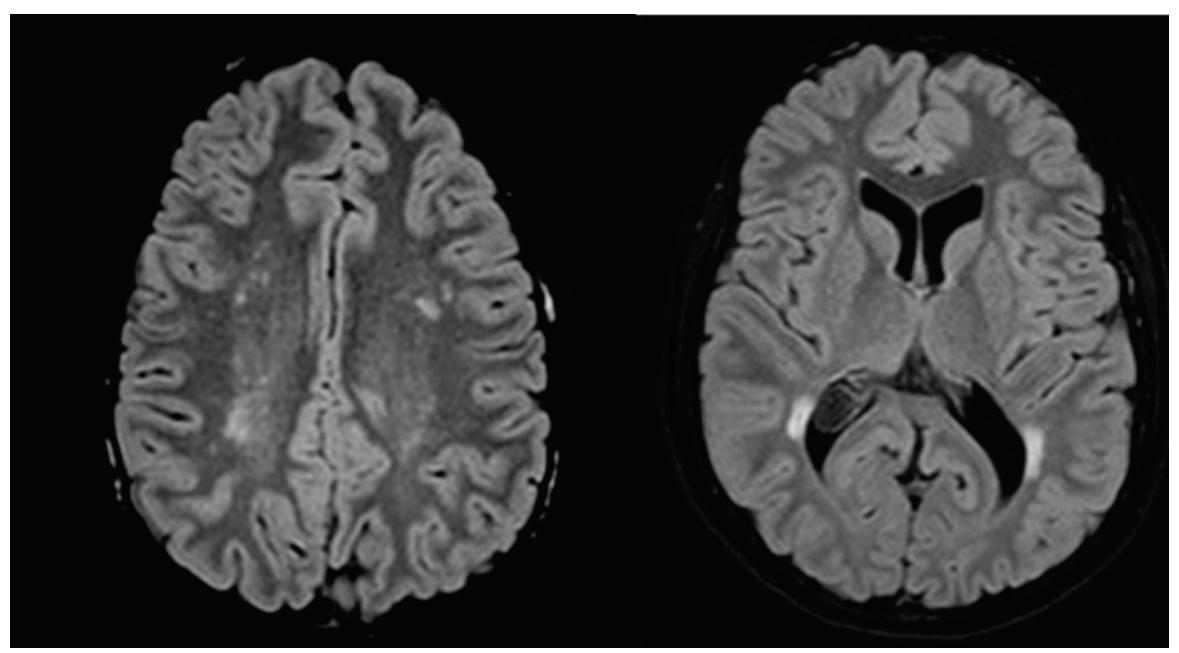

Figure 1. Axial FLAIR-MRI (1.5 T) reveals multiple T2 hyperintensities within the periventricular white matter of the frontal and parietal lobe, confluating in the peritrigonal area. No gadolinium enhancement or diffusion restriction was found.

Free $\gamma$-aminobutyric acid (GABA) and glutamate levels were measured in the patient's CSF (three times) and control samples from 10 subjects (five males and five females; age range 35 - 85 years (mean 55 years)) undergoing diagnostic lumbar puncture as part of their investigations. Their CSF analyses were normal and neurological disorders were excluded. The study was performed according to the Declaration of Helsinki and informed consent was obtained. All lumbar punctures were done between 11:00 am and 2:00 pm. Glutamate and GABA amounts of CSFs were determined by high performance liquid chromatography with electrochemical detection according to Prauss et al [3].

\section{Discussion}

The rate-limiting step in steroid biosynthesis is regulated by the steroidogenic acute regulatory protein (StAR) that mediates the transfer of cholesterol from the outer to the inner mitochondrial membrane [4]. Cholesterol is then converted by mitochondrial cytochrome P450scc, CYP11A1 to pregnenolone, the precursor for all steroids [1]. While the StAR mediated active process is available only in some tissues, pregnenolone synthesis is completely dependent on P450scc conversion. The brain synthesizes neurosteroids de novo, especially within glia [4]. Neurosteroids modulate function of several receptors, and regulate growth of neurons, synaptogenesis and especially myelination in the central nervous system (CNS) [5]. Deficits in neurosteroid production may contribute to a variety of disorders, including dementia and epilepsy [4]. Disruption of P450scc enzyme due to deleterious mutations of the CYP11A1 gene was thought to be incompatible with fetal survival because of impaired progesterone production by the fetoplacental unit. However, in the meantime two patients were described with homozygous disruption of CYP11A1 gene by Hiort et al [2] and Kim et al [6]. In one of them, as a child, complete 46,XY sex reversal and severe adrenal insufficiency were found [2]. In this currently 19-year-old patient, clinical neurological deficits could not be found; cerebral MRI, however, revealed multiple supratentorial T2 lesions without evidence of blood-brain barrier breakdown (Fig. 1). Since the lesions were especially periventricular and juxtacortical, they are comparable to those typically found in MS. According to the revised McDonald criteria [7], dissemination in space can be demonstrated with at least one T2 lesion in at least two of four locations considered characteristic for MS (juxtacortical, periventricular, infratentorial, and spinal cord). Interestingly, these authors proposed these criteria simple and sensitive for MS diagnosis without compromising specificity and accuracy [7].

Additionally, in the CSF, our present patient's GABA level was $13.0 \pm 0.3 \mathrm{nmol} / \mathrm{L}$ (from three measurements), lower than that in controls $(44.7 \pm 15.2 \mathrm{nmol} / \mathrm{L})$. Free glutamate level of the patient was $0.24 \mu \mathrm{mol} / \mathrm{L}$, slightly lower than that found in normal controls $(0.33 \pm 0.21 \mu \mathrm{mol} / \mathrm{L})$. Here the range of control GABA concentrations was in line with measurements of Piotrowicz and coworkers [8]. In MS patients, decreased levels of GABA, and also aspartate, glutamate, methionine and phenylalanine were found in CSF [9]. Also in single-voxel magnetic resonance spectroscopy, MS patients had significantly lower GABA levels in the hippocampus and sensorimotor cortex, which may suggest that reduced GABA levels reflect pathological abnormalities [10].

Myelin sheaths form during development and consist of compacted spiral wraps of membrane that are supplied by oligodendrocytes in the CNS and Schwann cells in the peripheral nervous system (PNS) [11]. Progesterone, produced by Schwann cells in response to their stimulation by adjacent neurons, regulates myelin protein synthesis via a nuclear progesterone receptor in the PNS [12]. In the CNS, oligodendrocytes were shown to express P450scc and also produce pregnenolone, progesterone and allopregnanolone [13]. In cerebellar slice cultures progesterone increased proliferation of oligodendrocyte precursors and promoted differentiation into mature cells [14]. Also in zebrafish model, especially radial glial cells were acting as neuronal progenitors and appeared to be a po- 
tential source of neurosteroids, but also as a target for centrally produced steroids [15]. The $3 \alpha$-reduced metabolites of progesterone are, additionally, potent positive allosteric modulators of $\mathrm{GABA}_{\mathrm{A}}$ receptors $[16,17]$. In the case of $\mathrm{P} 450 \mathrm{scc}$ disruption, this receptor modulation might be partially lost. Thus, we speculate that disturbance of $\mathrm{GABA}_{\mathrm{A}}$ receptor functioning might lead to a negative feedback inhibition of GABA release as described by Cryan and Kaupmann [18].

The MRI and CSF similarities between P450scc deficiency and MS let us point to the hypothesis published by Leitner [19]. The author hypothesized that etiology of MS with white matter pathology, axonal loss, failure of remyelination and the development of focal inflammatory lesions might be due to dysregulation of neurosteroid synthesis, leading to a disturbance of myelin maintenance and increased vulnerability of the myelin sheath, phagocytosis of myelin debris and processing of myelin antigen by microglia and leading to a complex and individually different immune response [19]. Although the precise etiology is indeed still unclear, MS is, however, commonly considered as a primary immunological-inflammatory disease probably triggered by genetic and environmental factors.

In conclusion, we describe a young patient with altered steroid metabolism due to a homozygous disruption of the $C Y$ $P 11$ Al gene. Since P450scc is crucial in steroidogenesis, we assume an impaired function of neurosteroidogenesis and myelin synthesis in the CNS leading to disease-related MRI and CSF neurotransmitter findings that are not diagnostic of MS.

\section{Abbreviations}

GABA: $\gamma$-aminobutyric acid; P450scc: P450 side-chain cleavage enzyme; CSF: cerebrospinal fluid; MRI: magnetic resonance imaging; MS: multiple sclerosis

\section{References}

1. Hauffa B, Hiort O. P450 side-chain cleavage deficiency - a rare cause of congenital adrenal hyperplasia. Endocr Dev. 2011;20:54-62.

2. Hiort O, Holterhus PM, Werner R, Marschke C, Hoppe U, Partsch CJ, Riepe FG, et al. Homozygous disruption of P450 side-chain cleavage (CYP11A1) is associated with prematurity, complete 46,XY sex reversal, and severe adrenal failure. J Clin Endocrinol Metab. 2005;90(1):538541.

3. Prauss K, Varatharajan R, Joseph K, Moser A. Transmitter self-regulation by extracellular glutamate in fresh human cortical slices. J Neural Transm (Vienna). 2014;121(11):1321-1327.

4. King SR, Manna PR, Ishii T, Syapin PJ, Ginsberg SD, Wilson K, Walsh LP, et al. An essential component in steroid synthesis, the steroidogenic acute regulatory protein, is expressed in discrete regions of the brain. J Neurosci. 2002;22(24):10613-10620.
5. Stoffel-Wagner B. Neurosteroid biosynthesis in the human brain and its clinical implications. Ann N Y Acad Sci. 2003;1007:64-78.

6. Kim CJ, Lin L, Huang N, Quigley CA, AvRuskin TW, Achermann JC, Miller WL. Severe combined adrenal and gonadal deficiency caused by novel mutations in the cholesterol side chain cleavage enzyme, P450scc. J Clin Endocrinol Metab. 2008;93(3):696-702.

7. Polman CH, Reingold SC, Banwell B, Clanet M, Cohen JA, Filippi M, Fujihara K, et al. Diagnostic criteria for multiple sclerosis: 2010 revisions to the McDonald criteria. Ann Neurol. 2011;69(2):292-302.

8. Piotrowicz A, Thumen A, Leite MI, Vincent A, Moser A. A case of glycine-receptor antibody-associated encephalomyelitis with rigidity and myoclonus (PERM): clinical course, treatment and CSF findings. J Neurol. 2011;258(12):2268-2270.

9. Qureshi GA, Baig MS. Quantification of free amino acids in biological samples by HPLC application of the method in evaluating amino acid levels in CSF and plasma of patients with multiple sclerosis. J Chromatogr A. 1988;459:237-244.

10. Cawley N, Solanky BS, Muhlert N, Tur C, Edden RA, Wheeler-Kingshott CA, Miller DH, et al. Reduced gamma-aminobutyric acid concentration is associated with physical disability in progressive multiple sclerosis. Brain. 2015;138(Pt 9):2584-2595.

11. Taveggia C, Feltri ML, Wrabetz L. Signals to promote myelin formation and repair. Nat Rev Neurol. 2010;6(5):276-287.

12. Plassart-Schiess E, Baulieu EE. Neurosteroids: recent findings. Brain Res Brain Res Rev. 2001;37(1-3):133140.

13. Zwain IH, Yen SS. Neurosteroidogenesis in astrocytes, oligodendrocytes, and neurons of cerebral cortex of rat brain. Endocrinology. 1999;140(8):3843-3852.

14. Ghoumari AM, Baulieu EE, Schumacher M. Progesterone increases oligodendroglial cell proliferation in rat cerebellar slice cultures. Neuroscience. 2005;135(1):47-58.

15. Diotel N, Do Rego JL, Anglade I, Vaillant C, Pellegrini E, Vaudry H, Kah O. The brain of teleost fish, a source, and a target of sexual steroids. Front Neurosci. 2011;5:137.

16. Wojtal K, Trojnar MK, Czuczwar SJ. Endogenous neuroprotective factors: neurosteroids. Pharmacol Rep. 2006;58(3):335-340.

17. Biagini G, Longo D, Baldelli E, Zoli M, Rogawski MA, Bertazzoni G, Avoli M. Neurosteroids and epileptogenesis in the pilocarpine model: evidence for a relationship between P450scc induction and length of the latent period. Epilepsia. 2009;50(Suppl 1):53-58.

18. Cryan JF, Kaupmann K. Don't worry 'B' happy!: a role for $\mathrm{GABA}(\mathrm{B})$ receptors in anxiety and depression. Trends Pharmacol Sci. 2005;26(1):36-43.

19. Leitner H. Influence of neurosteroids on the pathogenesis of multiple sclerosis. Med Hypotheses. 2010;75(2):229234. 